\title{
Elementary reduction of matrices over rings of almost stable range 1
}

\author{
B. Zabavsky, A. Romaniv, and T. Kysil \\ Communicated by V. V. Kirichenko
}

\begin{abstract}
In this paper we consider elementary reduction of matrices over rings of almost stable range 1 .
\end{abstract}

\section{Introduction}

One of important classes of rings that are widely used in modern algebra is the class of Euclidean rings. The existence of the Euclid algorithm is a great benefit for solving problems related to matrix calculations. Naturally, this fact is a reason of interest to study rings, such that every matrix over them can be reduced to the canonical diagonal form using only elementary operations. These rings are called the rings with elementary matrix reduction. However, there are elementary divisor rings that are not rings with elementary matrix reduction [4]. In the same time, as it will be shown in what follows, the use of elementary operations is crucial in elementary matrix reduction.

A ring is a right (left) Bezout ring if every finite generated right (left) ideal is principal.

Two matrices $A$ and $B$ over a ring $R$ are said to be equivalent if there exist invertible matrices $P$ and $Q$ such that $B=P A Q$. A matrix $A$ admits diagonal reduction if $A$ is equivalent to a diagonal matrix $D=\left(d_{i}\right)$ where $d_{i}$ is total divisor of $d_{i+1}$, i.e. $R d_{i+1} R \subset d_{i} R \cap R d_{i}$.

2010 MSC: 13F99.

Key words and phrases: elementary reduction of matrices, diagonal reduction of matrices, rings of almost stable range 1 . 
A ring $R$ is called an elementary divisor ring if every matrix over $R$ has a diagonal reduction [1].

We denote by $R_{n}$ the ring of all $n \times n$ matrices over $R$ and by $G L_{n}(R)$ its group of unites. We write $G E_{n}$ for the subgroup of $G L_{n}(R)$ generated by the elementary matrices. Denote by $(a, b)$ the greatest common divisor of elements $a, b$ of commutative ring $R$.

The Jacobson radical of a ring $R$ is denoted by $J(R)$.

A ring $R$ is called a ring of stable range 1 if for any elements $a, b \in$ $R$ the equality $a R+b R=R$ implies that there is some $x \in R$ such that $(a+b x) R=R$. If for any elements $a, b, c$ of a ring $R$ the equality $a R+b R+c R=R$ implies that there are some elements $x, y \in R$ such that $(a+c x) R+(b+c y) R=R$ then we say that the stable range is equal to 2 .

\section{The main results}

We need the following results to start.

Theorem 1 ([4]). Let $R$ be an elementary divisor ring. Then for any $n \times m$ matrix $A(n>2, m>2)$ one can find such invertible matrices $P \in G E_{n}(R)$ and $Q \in G E_{m}(R)$ that

$$
\left(\begin{array}{ccccccc}
\varepsilon_{1} & 0 & \ldots & 0 & 0 & \ldots & 0 \\
0 & \varepsilon_{2} & \ldots & 0 & 0 & \ldots & 0 \\
\ldots & \ldots & \ddots & \ldots & \ldots & \ldots & \ldots \\
0 & 0 & \ldots & \varepsilon_{s} & 0 & \ldots & 0 \\
0 & 0 & \ldots & 0 & & & \\
\ldots & \ldots & \ldots & \ldots & & A_{0} & \\
0 & 0 & \ldots & 0 & & &
\end{array}\right)
$$

where $\varepsilon_{i}$ is the total divisor of $\varepsilon_{i+1}, 1 \leqslant i \leqslant s-1$ and $A_{0}$ is $2 \times k$ or $k \times 2$ matrix for some $k \in N$.

Theorem 2 ([4]). Let $R$ be an elementary divisor ring. Then for any $n \times m$ matrix where $m-n=2$ there exist invertible matrices $P \in G E_{n}(R)$ and $Q \in G E_{m}(R)$ such that

$$
\left(\begin{array}{ccccccc}
\varepsilon_{1} & 0 & \ldots & 0 & 0 & \ldots & 0 \\
0 & \varepsilon_{2} & \ldots & 0 & 0 & \ldots & 0 \\
\ldots & \ldots & \ddots & \ldots & \ldots & \ldots & \ldots \\
0 & 0 & \ldots & \varepsilon_{s} & 0 & \ldots & 0 \\
0 & 0 & \ldots & 0 & 0 & \ldots & 0 \\
\ldots & \ldots & \ldots & \ldots & \ldots & \ldots & \ldots \\
0 & 0 & \ldots & 0 & 0 & \ldots & 0
\end{array}\right) .
$$


Definition 1. An element $a \neq 0$ of a commutative $\operatorname{ring} R$ is called an element of almost stable range 1 if the stable range of $R / a R$ is equal to 1 . If all nonzero elements of a ring $R$ are elements of almost stable range 1 , then we say that $R$ is a ring of almost stable range 1 .

The following results established in [5] play a key role in our work.

Proposition 1. Suppose $R$ is a commutative Bezout ring. If $R$ has stable range 1 , then $R$ is a ring of almost stable range 1 .

Theorem 3. Suppose $R$ is a commutative ring of almost stable range 1 . Whenever $a \notin J(R)$ and $a R+b R+c R=R$ there is $r \in R$ such that $a R+(b+c r) R=R$. In particular $R$ is a ring of stable range 2 .

Theorem 4. Suppose $R$ is a commutative ring of almost stable range 1 . Then $R$ is a Bezout ring if and only if $R$ is an elementary divisor ring.

By [6] we have a next result.

Proposition 2. Let $R$ be a commutative ring. The following properties are equivalent:

1) $R$ is a ring of almost stable range 1 ;

2) For any elements $a, b \in R$ such that $a R+b R+c R=R$ where $a \neq 0$ exist element $r \in R$ such that

$$
a R+(b+c r) R=R .
$$

Definition 2. A ring $R$ is said to be a ring of almost right(left) stable range 1 if for any elements $a, b, c \in R$ where $a \neq 0$ such that $a R+b R+c R=$ $R(R a+R b+R c=R)$ there exist an element $r \in R$ such that

$$
a R+(b+c r) R=R \quad(R a+R(b+r c)=R) .
$$

Open question. Is a ring of almost right(left) stable range 1 a ring of almost left(right) stable range 1 ?

Proposition 3. Let $R$ be a right(left) Bezout ring of stable range 1 then $R$ is of almost right(left) stable range 1 .

Proof. Let $a R+b R+c R=R, a \neq 0$ and $b R+c R=R$. Since $R$ is a ring of stable range 1 then $b=d b_{1}, c=d c_{1}$ where $b_{1} R+c_{1} R=R$ [4]. Then $\left(b_{1}+c_{1} \lambda\right) R=R$, i.e. $b_{1}+c_{1} \lambda=u$ - invertible element of $R$. Since $(b+c \lambda) R=d R$ and $a R+b R+c R=R$ then $a R+d R=R$ i.e. $a R+(b+c \lambda) R=R$. The proposition is proved. 
By [2] we have the following.

Proposition 4. Let $R$ be a commutative ring of almost stable range 1 . Then $R_{2}$ is a ring of almost right and left stable range 1 .

Theorem 5. Over a commutative Bezout domain of almost stable range 1 every $k \times(k+2)$ and $(k+2) \times k$ matrix, where $k \geqslant 2$, admits diagonal reduction by elementary transformations.

Proof. We prove the theorem by induction on the number of rows. Let $A$ be a $2 \times 4$ matrix. Without loss of generality we may change notation and assume that the greatest common divisor of all elements is 1 [4]. By Theorem 1, the matrix $A$ can be reduced by elementary transformations on the left to the form

$$
A_{1}=\left(\begin{array}{llll}
a & 0 & 0 & 0 \\
b & c & d & k
\end{array}\right)
$$

If $b=0$, by [4], the matrix $A$ can be reduced by elementary transformations to a diagonal form.

If $b \neq 0$, write $(a, b, c)=\delta, a=a_{0} \delta, b=b_{0} \delta, c=c_{0} \delta$ and $\left(a_{0}, b_{0}, c_{0}\right)=1$. Write $c_{0}=r s$ with the property asserted above. Then

$$
(r a+b, c)=(a, b, c)=\delta .
$$

Multiplying the first row of a matrix $A_{1}$ by $r$ and adding it to the second row, we obtain the matrix

$$
A_{2}=\left(\begin{array}{cccc}
a & 0 & 0 & 0 \\
r a+b & c & d & k
\end{array}\right)
$$

Since $(r a+b, c)=(a, b, c)$ and $(a, b, c, d, k)=1$, we have

$$
(r a+b, c, d, k)=1 .
$$

By [4], the matrix $A_{2}$ is reducible by elementary transformations to the form

$$
A_{3}=\left(\begin{array}{cccc}
1 & 0 & 0 & 0 \\
* & * & * & *
\end{array}\right) .
$$

It is obvious that $A_{3}$ is reducible by elementary transformations to a diagonal form. Induction on the number of rows completes the proof. 
Theorem 6. Let $R$ be commutative Bezout domain of almost stable range 1 , then for any nonsingular matrix of size $n$ we can find such unimodular matrices $P \in G E_{n}(R)$ and $Q \in G L_{n}(R)$, that

$$
P A Q=\left(\begin{array}{cccc}
\varepsilon_{1} & 0 & \ldots & 0 \\
0 & \varepsilon_{2} & \ldots & 0 \\
\vdots & \vdots & \ddots & \vdots \\
0 & 0 & \ldots & \varepsilon_{n}
\end{array}\right),
$$

where $\varepsilon_{i}$ is divisor $\varepsilon_{i+1}, 1 \leqslant i \leqslant n-1$.

Proof. Since commutative Bezout domain of almost stable range 1 is elementary divisor domain (see Theorem 4), then, it is obvious, by Theorem 1, to prove the statement it is sufficient to consider the case of a matrix of size 2. Without loss of generality, we can suppose, that the greatest common divisor of elements of the matrix equal 1 [4]. Since $R$ is Hermite ring, then by corollary 2.1.1 [4] stable range of $R$ is less or equal to 2 . Then for any matrix $A$ we can find a matrix $Q_{1} \in G L_{2}(R)$ such that

$$
A Q_{1}=\left(\begin{array}{ll}
a & 0 \\
b & c
\end{array}\right)
$$

Note, that

$$
a R+b R+c R=R .
$$

Since $A$ ia nonsingular, then $a \neq 0$ and $c \neq 0$. Due to the fact that $R$ is commutative Bezout domain of almost stable range 1 ,

$$
(r a+b) R+c R=R .
$$

If we multiply the first row of $A Q_{1}$ by $r$ and add to the second row of $A Q_{1}$, we obtain the matrix

$$
\left(\begin{array}{cc}
a & 0 \\
r a+b & c
\end{array}\right)
$$

Since

$$
(r a+b) R+c R=R,
$$

then exists a unimodular matrix $Q_{2} \in G L_{2}(R)$ such that

$$
\left(\begin{array}{cc}
a & 0 \\
r a+b & c
\end{array}\right) Q_{2}=\left(\begin{array}{cc}
* & * \\
1 & 0
\end{array}\right)
$$


Obviously, the matrix

$$
\left(\begin{array}{cc}
a & 0 \\
r a+b & c
\end{array}\right) Q_{2}
$$

can be reduced via elementary operations to

$$
\left(\begin{array}{cc}
1 & 0 \\
0 & a c
\end{array}\right) .
$$

\section{References}

[1] Kaplansky I. Elementary divisors and modules. Trans. Amer. Math. Soc. 166, 1966, pp.464-491.

[2] Shchedryk V. Bezout Rings of Stable Range 1.5 Ukrainian Math. J., 67 (6), 2015, pp.960-974.

[3] Zabavsky B. Reduction of matrices over right Bezout rings with finite stable rank. Matematychni Studii, 16 (2), 2001, 115-116.

[4] Zabavsky B. Diagonal reduction of matrices over rings. Mathematical Studies, Monograph series, vol. XVI, VNTL Publishers 2012, 249p.

[5] McGovern W. Bezout rings with almost stable range 1 are elementary divisor rings. J. Pure and Appl. Algebra, 212, 2007, pp.340-348.

[6] Anderson D., Juett J. Stable range and almost stable range. J. Pure and Appl. Algebra, 216, 2012, pp.2094-2097.

\section{CONTACT INFORMATION}

Bohdan Zabavsky Ivan Franko National University of L'viv, 1 Universytetska str.,79000, L'viv, Ukraine E-Mail(s): zabavskii@gmail.com

Andriy Romaniv Pidstryhach Institute for Applied Problems of Mechanics and Mathematics NAS of Ukraine, 3b Naukova Str., L'viv, 79060, Ukraine E-Mail(s): romaniv_a@ukr.net

Tatyana Kysil

Khmelnytskyi National University, 11 Institutska str., 29000, Khmelnitsky, Ukraine E-Mail(s): kysil_tanya@ukr.net

Received by the editors: 04.07.2018. 